NOTICIAS Y COMENTARIOS 



\title{
LA ORDENACIÓN TERRITORIAL EN EL PAÍS VASCO. DEBILIDADES, AMENAZAS, FORTALEZAS Y OPORTUNIDADES DE LA ESCALA INTERMEDIA
}

\author{
Pedro J. Lozano Valencia \\ Departamento de Geografía, Prehistoria y Arqueología \\ Universidad del País Vasco
}

\section{RESUMEN}

La ordenación del territorio encuentra su razón de ser, en el País Vasco, a través de las Directrices de Ordenación y dos tipos diferentes de documentos: los Planes Territoriales Parciales y los Planes Territoriales Sectoriales. Una vez delimitadas las comarcas y los sectores estratégicos, se aplican dichos documentos. Con todo, se logra la coordinación de las Directivas europeas a escala superior, y los Planes y Normas Subsidiarias de Ordenación Urbana a una escala inferior o local. En medio quedan todas esas herramientas de ordenación mencionadas anteriormente, que conforman lo que se ha venido a definir como la escala intermedia. Ésta aparece como un marco muy apropiado para abordar las debilidades, amenazas, fortalezas y oportunidades que, en un horizonte de 16 a 20 años, presenta el territorio vasco.

Palabras clave: Directrices de Ordenación, Planes Territoriales Parciales, Planes Territoriales Sectoriales, escala intermedia, debilidades, amenazas, fortalezas y oportunidades.

\begin{abstract}
Land use management in the Basque Country. strengths, weaknesses, opportunities and threats at an intermediate level. Land use management in the Basque Country is based on Land Use Management Guidelines and two different types of documents: Partial Territorial Plans and Sectorial Territorial Plans. Once the regions and strategic sectors have been delimited, these documents are applied. As a result, coordination of the European guidelines is achieved on a superior level and that of Land Use Management Subsidiary Planning and Norms on an inferior or local level. In between these levels are the rest of those management tools that have been mentioned previously and which constitute the group which has been defined as the intermediate level. This level forms an extremely effective framework for dealing with the strengths, weaknesses, opportunities and threats that, within a period of 16 to 20 years, may exist in the Basque Country.
\end{abstract}


Key words: Land Use Management Guidelines, Partial Territorial Plans, Sectorial Territorial Plans, intermediate level, strengths, weaknesses, opportunities and threats.

\section{Introducción}

Se puede definir la Ordenación del Territorio como la representación en el espacio de las decisiones políticas en materias tan diferentes como: sociedad, economía, cultura, medio ambiente, etc. Esta definición vendría a ser muy similar a la propuesta por la Carta Europea de Ordenación Territorial. También sería la base de diferentes trabajos científicos (ZOIDO 1998). Pero a esta definición se le deberían añadir una serie de acotaciones que nos dieran una idea más clara de lo que se puede entender por ordenación territorial (O.T.). La primera característica es la de constituirse como una disciplina relativamente nueva, con unos contenidos bastante amplios y discutidos, puesto que, en $2^{\circ}$ lugar, habría que reseñar que son muchos los agentes y profesionales que toman parte en el proceso de ordenación y gestión del territorio. En este sentido, cabe destacar que estamos ante una ocupación, por definición, ciertamente multidisciplinar.

La ordenación del territorio debe contar con esta multidisciplinaridad y carácter general puesto que el proceso implica, de forma global, a todos los agentes, procesos y relaciones que aparecen sobre el espacio. De esta manera, mientras para los geógrafos el territorio es la herramienta de trabajo y los diferentes agentes no son sino atributos del mismo, cada una de las parcelas profesionales o del conocimiento implicadas en el proceso de ordenación, analizaran y diagnosticaran el territorio pero caracterizándolo a través del agente propio de estudio. No obstante, cada una de estas informaciones sectoriales, deben ser tenidas en cuenta y valoradas en su justa medida de manera que, partiendo de un trabajo de síntesis ciertamente dificultoso y meritorio, se de lugar a la elaboración de las alternativas necesarias para hacer frente a los problemas y promover el desarrollo de las potencialidades existentes.

Además de estas cuestiones, el carácter global de la O.T. se pone de manifiesto al tener en cuenta que supone la planificación a futuro de todos los aspectos citados con anterioridad: sociedad, medio ambiente, economía, cultura, etc. Así, se configura como el marco previo y coordinado, desde el que desarrollar cada una de las políticas sectoriales. La O.T., por lo tanto, afecta a todas las actuaciones públicas con incidencia territorial, dándoles un tratamiento integrado (PUJADAS y FONT 1998).

La elaboración de estos planes pasa por ser uno de los trabajos profesionales más complicados. Abordar el territorio desde un prisma global y para la buena y racional gestión de todos los recursos existentes, lleva consigo la necesidad de poner en marcha una metodología ciertamente pormenorizada y complicada, puesto que mucha es la responsabilidad a la hora de aconsejar y poner en marcha o ejecutar las políticas necesarias para la buena consecución de este primer objetivo. Por ello, partiendo de un análisis pormenorizado de cada uno de los atributos, se debe llegar a un diagnóstico integrado que nos informe, de la manera más fidedigna posible, de la situación del territorio en cuestión. Una vez obtenido el diagnóstico, se debe actuar como lo haría un médico ante su paciente; de forma global, poniendo remedio a los males y haciendo hincapié en las potencialidades. A todo ello, se debe añadir el carácter prospectivo, ya que se planifica para el futuro, el carácter abierto o democrático, puesto que en este proceso debe ser tenida en cuenta la opinión y valoración de la sociedad, así como el carácter consultivo que estos documentos deben presentar, de cara a la toma de decisiones por parte de las autoridades políticas competentes. 
Centrándonos en el País Vasco, la ordenación del territorio cuenta con un antecedente claro. Mientras hasta la cesión de las competencias en esta materia al Gobierno de la Comunidad Autónoma Vasca, la ordenación territorial se realizaba a través del cumplimiento de la ley sobre el régimen del suelo y la ordenación urbana, a partir de 1979, mediante la denominada vulgarmente: Ley de Territorios Históricos del País Vasco, en realidad Ley orgánica 3/1979, de 18 de diciembre, Estatuto de Autonomía del País Vasco, por el cual; como anteriormente se ha comentado, existe una transferencia competencial, entre las que se encuentra la de ordenar y gestionar el territorio, dependerá de las autoridades autonómicas, de manera que al respecto se da lugar a una ley marco como es la de Ordenación del Territorio en el País Vasco. En realidad, el enunciado de esta ley es el siguiente: Ley 4/1990 de 31 de mayo, de Ordenación del Territorio del País Vasco. Junto a esta o, partiendo de ésta, se elabora otra que ya realiza un acercamiento más pormenorizado a la regulación que debe alumbrar la gestión del territorio en esta Comunidad Autónoma. Este documento legal es conocido vulgarmente como las DOT, en realidad, un apocope de: Decreto 28/1997, de 11 de febrero por el que se aprueban definitivamente las Directrices de O.T. de la Comunidad Autónoma del País Vasco.

Este último código legal, ciertamente interesante, regula de forma exhaustiva como debe ser la Ordenación del Territorio, de manera que partiendo de unas líneas generales, genera una comarcalización, que ha ido fluctuando dependiendo del desarrollo, tanto de las políticas, como de las propias comarcas, y apunta las líneas básicas para cada una de estas unidades territoriales. También regula cuales deben ser los documentos de ordenación que deben ser puestos en marcha, así como sus plazos de renovación y revisión.

Por último, haciendo referencia a un concepto apuntado en el título del presente artículo, la escala intermedia, nombre cuyo momento de acuñación escapa al conocimiento del presente autor, representa, a nuestro modo de ver, una magnífica definición para aquella escala de trabajo que se encuentra entre las directivas europeas y las legislaciones de competencia estatal; como aquellas de escala mayor o superior, y las de escala inferior o local; caracterizadas por los planes y normas subsidiarias de ordenación urbana.

Hasta hace muy poco tiempo, las directrices generales existían, pero sólo contaban con un hito ejecutivo; cada uno de los planes puestos en marcha a nivel local o municipal. En este sentido, se echaba en falta un documento que aunara estos dos niveles de ordenación tan profundamente separados, tanto en la escala, como, muchas veces, en el espacio. Por ello, creemos que ordenación del territorio y escala intermedia son dos conceptos que pueden ser tan semejantes que, en el futuro, puedan ser considerados como sinónimos. De hecho, en el presente y en el territorio que nos ocupa, son tomados de forma paralela y desarrollados a la misma vez.

\section{Diferentes figuras de planificación dentro de la escala intermedia en el País Vasco}

En realidad, hace un tiempo, el famoso literato Bernardo Atxaga, de una forma poética y retórica, acuñó el nombre de la ciudad vasca «Euskal-hiria» como un todo, formado por diferentes partes que, sin embargo, perdería su entidad y su vida si dichas partes fueran disociadas. Dicho concepto parece ajustarse perfectamente a la realidad del propio territorio vasco. Nos encontramos ante una comunidad autónoma muy reducida en espacio $\left(7.482 \mathrm{~km}^{2}\right)$, que se reparten de la siguiente manera: Álava $\left(3.268 \mathrm{~km}^{2}\right)$, Vizcaya $(2.217$ $\mathrm{km}^{2}$ ) y Guipúzcoa (1.977 km²), y que exceptuando las comunidades uniprovinciales y las formadas por archipiélagos isleños, se puede considerar como la más pequeña del estado español. A su vez, las competencias, aunque la mayoría son entregadas por el poder central a la propia comunidad, como consecuencia de la anteriormente citada ley de territorios 
históricos, y por la tradición peculiar y foralista de esta región, en gran medida, son absorbidas por las propias Diputaciones Forales. De esta forma, tanto éstas, como el Gobierno Vasco, deben coordinar el desarrollo de las leyes y los planes necesarios para la ejecución de la ordenación del territorio.

El concepto de ciudad vasca se acerca al acuñado a mitad de siglo pasado; «CiudadRegión», de manera que, en este espacio tan reducido, es necesario coordinar y aunar las políticas encaminadas a la O.T. Dicha coordinación viene reflejada ya en las directrices de ordenación, aunque no se debe perder de vista la realidad de las diferentes comarcas, también bien definidas por este mismo código legal.

Teniendo en cuenta estos conceptos previos, el Gobierno Vasco y las diferentes Diputaciones Forales ponen en marcha dos tipos de documentos bien diferenciados, que dan lugar a la planificación, tanto de las comarcas como de los sectores. Por ello, se procede a la licitación y ejecución de los trabajos encaminados a generar los Planes Territoriales Parciales (PTP), para cada una de las comarcas, y los Planes Territoriales Sectoriales (PTS), para cada uno de los sectores.

Aunque las DOT y estos dos tipos de planes ya habían sido contemplados y licitados con anterioridad, no habían podido, en la mayoría de los casos, ni llegar a ver la luz, como consecuencia de ciertas cuestiones entre las que cabría destacar la no suficiente toma en cuenta de su importancia, la imposibilidad de abordar de forma global ciertos territorios y sectores con competencias repartidas o atomizadas, así como el excesivo poder de las administraciones locales (municipios), y sus planes de ordenación urbana.

Con todo, hoy en día existe una fuerte irrupción de los planes parciales de cada una de las comarcas, así como los planes sectoriales de cada uno de los sectores. Dicha irrupción no quiere decir que se hayan solventado los problemas apuntados en el párrafo anterior, sino que hacen patente la necesidad de generar estos documentos, de cara a un futuro incierto, donde la ordenación territorial a esta escala intermedia parece un requisito obligatorio para el desarrollo de la Comunidad Autónoma Vasca. La competencia entre las distintas regiones que integran la Unión Europea es palpable, y aquellas que no desarrollen los suficientes planes y las directrices de desarrollo, de una manera lógica y estudiada de antemano, con estrategias muy consolidadas, deberán atenerse a la situación más favorable de aquellas que si hicieron los deberes en su momento.

En este sentido, el País Vasco parte como la comunidad o región de España donde más rapidamente se está haciendo frente a la O.T. a estas escalas intermedias. No es cierto que los planes antes apuntados no existan en otras autonomías, de hecho, aunque con diferentes nombres, hay regiones que sí los contemplan: Andalucía con sus planes subregionales y Cataluña con sus Planes de Choque, por poner dos ejemplos, sino que el País Vasco, cuenta con un desarrollo más adelantado de los Planes Parciales (todos están en proceso) y con un buen número de Planes Sectoriales.

A continuación se detallan dichos documentos y sus diferentes tipos:

\subsection{Los planes territoriales parciales (PTP)}

De Acuerdo con la ley de ordenación del territorio del Gobierno Vasco, los planes territoriales parciales deberán desarrollar lo apuntado por las directrices de ordenación dentro de cada una de las áreas funcionales delimitadas. Éstas son especificadas por las propias DOT. Dicha delimitación se ha basado en una serie de criterios geográficos, económicos y sociales. En realidad se trata de áreas bastante homogéneas, bien diferenciadas del resto y con un funcionamiento determinado, fundamentalmente a nivel de áreas de atracción y red de asentamientos. También juegan un papel fundamental cuestiones pura- 
mente históricas y geográficas, de hecho, no es poca la importancia que ostentan estas dos dimensiones a la hora de delimitarlas. Hay que tener en cuenta que las delimitaciones fueron apuntadas al comienzo del funcionamiento de la autonomía por el Instituto Geográfico Vasco Andrés de Urdaneta (INGEBA).

Cada uno de los PTP contiene las siguientes determinaciones:

A) La definición de los objetivos de la ordenación a partir del análisis del estado actual del territorio, de la situación socioeconómica y de sus posibilidades de evolución.

B) El señalamiento de los espacios óptimos para servir de soporte a las grandes infraestructuras, según sus características.

C) La definición de la ubicación de los equipamientos de interés común para el área o zona objeto del plan.

D) Los criterios, principios y normas generales a los que habrá que atenerse la ordenación urbanística.

E) La definición de los espacios que deban ser objeto de remodelación, regeneración o rehabilitación con el fin de evitar su degradación o de conseguir su recuperación para usos, total o parcialmente distintos, así como de los programas a desarrollar y las medidas de apoyo encaminadas a incentivar su realización.

F) La cuantificación de las superficies de suelo que deban reservarse con destino a algunas de las siguientes finalidades:

- Construcción de viviendas de protección oficial, de promoción pública o priva$\mathrm{da}, \mathrm{u}$ otras que en el futuro pudieran ser limitadas en su precio final mediante regulación específica.

- Promoción pública de suelo industrial para posibilitar la formación de polígonos urbanizados.

G) Los criterios, normas y principios necesarios para el desarrollo de las determinaciones que contienen las directrices.

Además, hay que tener en cuenta las cuestiones que no son competencia directa de un PTP. Entre éstas, habría que destacar que no califica ni clasifica el suelo. Es decir, no cuenta con las competencias que le lleven a determinar las diferentes clases del suelo que, según la ley que regula el mismo, serian urbanizado, urbanizable y no urbanizable. La calificación a partir de estas figuras, además de la clasificación de este recurso, sigue recayendo en los entes locales, en los municipios. Los planes que se encargan de ello son los planes generales y las normas complementarias o subsidiarias municipales de ordenación. Lo que pretenden ser es documentos de obligada consulta a la hora de redactar los planes de carácter local.

Los planes territoriales parciales tampoco deben ser la suma de una serie de planes urbanísticos, sino que deben contar con personalidad propia y no configurarse como un mero sumatorio de planificaciones municipales distintas.

Por otra parte, estos PTP deben concretar sus determinaciones a través de los siguientes documentos:

A) Estudios y planos de información.

B) Memoria explicativa del plan.

C) Estudio económico-financiero.

D) Programa de ejecución, desglosado en etapas de 4 años.

E) Planos y normas de ordenación.

F) Otros documentos que el redactor pueda considerar necesarios o adecuados. 
Teniendo en cuenta lo anteriormente expuesto, se han determinado las siguientes áreas funcionales y, por tanto, los preceptivos Planes Territoriales Parciales:

Para Vizcaya existen 6 áreas funcionales diferenciadas de la siguiente manera:
A) Área funcional de Balmaseda-Zalla (englobando 10 municipios).
B) Área funcional del Bilbao metropolitano (englobando 33 municipios).
C) Área funcional de Durango (englobando 11 municipios).
D) Área funcional de Gernika-Markina (englobando 30 municipios).
E) Área funcional de Igorre (englobando 9 municipios).
F) Área funcional de Mungia (englobando 9 municipios).

Para Guipúzcoa existen otras 6 áreas funcionales diferenciadas de la siguiente manera:
A) Área funcional de Beasain-Zumarraga (englobando 24 municipios).
B) Área funcional de Donostialdea-Bajo Bidasoa (englobando 13 municipios).
C) Área funcional de Eibar (englobando 8 municipios).
D) Área funcional de Mondragon-Bergara (englobando 9 municipios).
E) Área funcional de Tolosaldea (englobando 27 municipios).
F) Área funcional de Zarautz-Azpeitia (englobando 10 municipios).

Para Álava existen 3 áreas funcionales diferenciadas de la siguiente manera:
A) Área funcional de Laguardia (englobando 15 municipios).
G) Área funcional de Llodio (englobando 8 municipios).
H) Área funcional de Álava central o Vitoria-Gasteiz (englobando 35 municipios).

En realidad tampoco se respetan los límites administrativos provinciales a la hora de delimitar áreas funcionales, no de una forma extremadamente escrupulosa. De esta manera, municipios como Ermua, dentro de territorio vizcaino, se encuentran dentro de una realidad funcional que tiene más que ver con ciertos núcleos del territorio guipuzcoano y, por ello, es englobada dentro del área de Eibar, de hecho, este territorio puede ser definido como área funcional Eibar-Ermua puesto que son los dos grandes núcleos que la dominan y que forman un continuo. También municipios como Orozco se encuentran dentro de territorio vizcaino aunque mantienen una funcionalidad más ligada a núcleos más importantes pero alaveses como Amurrio o Llodio, aunque estos últimos mantienen una mayor relación e interdependencia con Bilbao de la que mantienen con la propia Vitoria. Como se observa, estos ejemplos son relativamente abundantes aunque lejos de considerarse como un handicap no hacen sino reflejar una realidad funcional que se ha tenido en cuenta de una forma bastante acertada.

En la actualidad la redacción de los 15 planes se halla en diferentes fases, aunque todos ellos se encuentran iniciada esta fase de redacción. Así, ya pueden ser consultados los avances de los planes territoriales parciales de diferentes áreas como: el Bilbao metropolitano, Álava central, Gernika-Markina, etc.

Cada uno de los PTP cuenta con un carácter propio derivado, no sólo de las peculiaridades del territorio de estudio, sino de las propias características del equipo de trabajo que lo ha abordado. En realidad existen dos grandes estrategias, una más desarrollista y otra más medioambientalista o de protección, también existe una gran cantidad de matices entre 
estos dos extremos, pero, sin duda, aparece un sesgo claro dependiendo de los equipos redactores.

A continuación se detallan, de forma sucinta, 2 ejemplos de planes territoriales parciales de 2 áreas muy diferentes. En este sentido, hay que destacar la diferencia general entre las áreas más densamente urbanizadas como: el Bilbao metropolitano, Donostialdea o el área de San Sebastián y la zona de Vitoria-Gasteiz, y aquellas que mantienen todavía cierto carácter rural y un suelo menos densamente urbanizado; el resto.

\subsubsection{P.T.P. Área funcional metropolitana de Bilbao}

Este plan territorial parcial comienza haciendo referencia a la necesidad de realizar este tipo de planes no solamente por obligación legal, sino debe ser palpable por la sociedad la necesidad de su realización y ejecución. Que sea algo sentido no sólo por la población, sino por los diferentes ayuntamientos que forman la comarca a ordenar. Estos PTP además deben ser generales y abarcar y vincular a todos los aspectos o factores que actúan en el territorio. Hay que hacer una salvedad; las carreteras cuentan con su propia línea y desarrollan su propio PTS.

Los planes deben elegir y seleccionar donde se ubican algunas cuestiones de gran tamaño, no partibles, no divisibles. Estas son aquellas infraestructuras, servicios, equipamientos que son de todos y para todos, pero que sólo pueden situarse en un sitio. Quizás estos planes sean la herramienta más efectiva y aséptica de análisis y ubicación para este tipo de localizaciones.

En lo que respecta al área que nos ocupa, el Gran Bilbao cuenta con unas características bien particulares; se termina con un modelo industrial de ocupación indiscriminada y una industria pesada y contaminante, con un puerto continuo, una ría cloaca, un urbanismo exagerado, etc. y se lucha por superar eso a través de la reconversión de suelos hacia unos usos más acordes con las nuevas perspectivas sociales y económicas del área metropolitana. El efecto Gugemheim ha promovido más la ruina de la fortaleza industrial y la reconversión hacia nuevos usos. El aparente problema inicial de la reconversión se ha convertido en un verdadero éxito.

La idea clave, así pues, es reciclar el suelo, el terminar con la huida hacia nuevos valles, la colonización de nuevos suelos mientras los ya consolidados pierden la funcionalidad para la que fueron creados. Además de esto, la zona más afectada por la obsolescencia de usos, que es el centro de la ría, debe configurarse como el verdadero eje de desarrollo de las nuevas ideas. La ría, por lo tanto, aparece como el lugar estratégico donde se concentra la actuación del PTP.

Otra de las actuaciones básicas es el traslado del Campo de Fútbol de San Mames y la Feria de Muestras. Estos dos hitos se están configurando como un verdadero tapón al crecimiento y desarrollo del área metropolitana.

Para que todo este espacio tenga fuerza hay una serie de cuestiones que son irrenunciables. Sin embargo, la realidad es que algunas no se están realizando con lo que se está perdiendo un tiempo estratégico precioso.

Una de las que se está abordando es el pasar el puerto lineal al exterior. Con ello, se trasladan los usos que más superficie necesitan fuera de este espacio y se recuperan unas parcelas realmente extensas, además se puede realizar el cosido de las dos márgenes de la ría, de manera que la margen derecha y la izquierda puedan ser unidas con nuevos puentes y líneas de infraestructura.

De la misma forma, se requiere un profundo cambio en el sistema de transportes. Este se ha realizado generando un sistema de transporte colectivo ferroviario como es el 
metro de Bilbao. También se toma como necesario, y no se está realizando, que se desmantele el ferrocarril de vía ancha del lugar que ocupa y sea alejado del centro de la ría. Este trazado ferroviario también está hipotecando, en parte, el desarrollo del área metropolitana.

Por último, se requiere una eficaz limpieza de la ría, cuestión que ya se viene realizando pero que debe ser más profunda y abarcar algunos aspectos más.

El PTP, se ha centrado en aquellas cuestiones o acciones positivas, de permitir cosas e intentar prohibir lo menos posible. Para ello se han tenido en cuenta dos tipos de intervenciones:

- Acciones estucturantes: Destinadas a otorgar una estructura, sobre todo de comunicaciones, a la metrópoli.

- Acciones estratégicas: Operaciones sobre parcelas concretas, acciones puntuales para potenciar el carácter metropolitano del área del Gran Bilbao.

La característica fundamental de este PTP es que según se va realizando se van ejecutando acciones, a veces a gran velocidad. Desgraciadamente esto es algo que debe ocurrir en estas áreas tan dinámicas pero, afortunadamente, puede presentar buenos resultados cuando las estrategias y líneas a seguir son claras y todos los agentes tienen en cuenta los objetivos generales y globales.

Para reforzar el carácter central de la ría se ha dado lugar a la existencia de toda una serie de infraestructuras de conexión que se traducen en el metro, en un tranvía de superficie articulado y ligero y en una serie de líneas diferentes de transportes colectivos por autobús.

\subsubsection{PTP Área funcional de Gernika-Markina (Urdaibai)}

La comarca que nos ocupa se encuentra fuera de los grandes ejes de comunicación, pero esto, lejos de ser un aspecto negativo, se puede convertir en una oportunidad. La potencialidad de esta idea viene de manos del desarrollo sostenible. En este sentido, dentro del presente plan se hace referencia a un documento que ha servido de base para su realización. Se trata de: Principios para una estrategia de desarrollo sostenible en el territorio (Posdam 1999).

El área que nos ocupa cuenta con un valor estratégico como corazón verde del conjunto del País y de equilibrio territorial rural y ambiental.

Se trata de un territorio producto de la ocupación ancestral del ser humano, intensamente cambiado precisamente por éste y por una actividad además agropecuaria.

Aquí además, las oportunidades vienen de mano de los abundantes espacios naturales bien conservados y gestionados.

La estrategia fundamental es el mantenimiento de una agricultura y ganadería capaz de sostener, de una manera viva y no como producto de museo, este paisaje. Un modelo de asentamiento policéntrico.

Otras características de este territorio son:

- Espacio con graves desequilibrios.

- Dinámica demográfica regresiva.

- El caserío como núcleo productivo y tipo de explotación en clara y rápida decadencia.

- Zona donde los riesgos sobre el territorio son muy evidentes y las fragilidades son realmente altas. 
- Territorio intensamente desvertebrado con infraestructuras muy mal diseñadas y que además se han quedado obsoletas.

- Es un área que incluso no logra dar empleo suficiente a la escasa población residente.

- La perspectiva demográfica inmediata es de desequilibrio inminente, de hecho, el mantener este territorio va a significar un esfuerzo realmente notable.

- La dualidad entre la costa y el interior es buena y positiva. En el interior existen una serie de montes y valles donde se distribuyen centros urbanos a veces de conjuntos de caseríos, otras veces con núcleos más importantes y donde los espacios agropecuarios están siendo sustituidos gradualmente por el monocultivo maderero. El litoral, sin embargo, cuenta con unos valores de atracción del turismo realmente notables.

En lo que respecta al modelo territorial propuesto, este se resume en los puntos:

- El área funcional de Gernika-Markina constituye una pieza clave para el equilibrio ambiental y rural del País Vasco.

- Fortalecimiento del modelo urbano policéntrico potenciando las relaciones de complementariedad entre los núcleos.

- La apuesta es la buena infraestructuración del territorio, lo cual no tiene por que llevar pareja la urbanización.

- Preservar el patrimonio y la filosofía de los núcleos tradicionales.

- El turismo es una oportunidad, pero no debe ser canalizado a través del chalet de segunda residencia.

- Modernización del tejido productivo que pasa por regenerar y rehabilitar los polígonos ya hechos y actuaciones nuevas inevitablemente públicas.

- En cuanto a las infraestructuras: potenciar las grapas o vínculos del territorio con los vecinos. Interesa vincular los diferentes núcleos a través de ejes ya consolidados pero manifiestamente mejorables.

- La conservación o utilización creativa de los recursos naturales y el paisaje. El punto crítico son las campiñas porque los montes ya se encuentran defendidos. Cuidado con la diseminación incontrolada de la vivienda de baja densidad.

- La Reserva de la Biosfera puede funcionar como un elemento positivo de potenciación endógeno.

- Hay que mejorar la accesibilidad exterior y no la interior. No hay que tender a rellenar el interior del anillo, sino el exterior, muy en la línea del ejemplo del Ranstad holandés.

- Existen cinco núcleos esenciales: Gernika-Lumo, Markina, Bermeo, Lekeitio y Ondaroa. Es Markina el núcleo que debe ir ganando peso con un crecimiento controlado.

- A nivel ambiental se debe apostar por el patrocinio de la zona a través de:

- Unas rutas naturales y ambientales de carácter turístico.

- La conservación de áreas rurales de interés territorial.

- La recuperación de canteras y áreas impactadas, etc.

Por su parte, el PTP se centra mucho en conservar las zonas de campiña y para ello propone el siguiente criterio:

- El punto fundamental es facilitar la pervivencia de la actividad agraria. Para ello deben tenerse muy en cuenta actividades de agricultura y ganadería que apuesten 
por la calidad. También pueden llevarse a cabo complementos como casas rurales de agroturismo, actividades culturales y medioambientales, etc.

La conservación de las campiñas, como se puede comprobar, se configura como un objetivo prioritario, frente a la presión por este suelo que ejerce la vivienda de esparcimiento o segunda residencia y la que, aún siendo de primera residencia, apuesta por modelos de baja densidad.

\subsection{Planes territoriales sectoriales (PTS)}

Lo primero a reseñar es la existencia, a nivel de OT, de tres instrumentos légales básicos a nivel del País Vasco. El primero es la Ley de OT del año 1990, el segundo es la Ley de conservación de la Naturaleza del 1994 y, para finalizar, en tercer lugar se encuentra la Ley de Medio Ambiente de 1998. Estos tres hitos legislativos van a ser los que van a conformar la realización de los preceptivos documentos de ordenación.

Hay que destacar que existen tres áreas bien definidas a nivel de PTS: La $1^{\text {a }}$ hace referencia a la ejecución de la política de suelo. La $2^{\underline{a}}$ a las grandes infraestructuras y la $3^{\underline{a}}$ a la política en medio ambiente.

El objetivo fundamental de todos estos Planes Territoriales Sectoriales es la regulación de los diferentes instrumentos de ordenación sectorial de las administraciones vascas.

En la actualidad se han definido veinticinco planes territoriales sectoriales, de los cuales cuatro han sido aprobados:

- PTS de Ordenación de las márgenes de ríos y arroyos.

- PTS de Carreteras de la C.A.V.

- PTS de Carreteras de Álava.

- PTS de Carreteras de Vizcaya.

Otros ocho se encuentran en diferentes fases de realización:

- PTS de Infraestructura ferroviaria.

- PTS de Ordenación de las zonas húmedas.

- PTS de ordenación del litoral.

- PTS de Energía eólica.

- PTS de Patrimonio cultural.

- PTS Agroforestal.

- PTS de Creación de suelo para actividades económicas.

- PTS de Creación de suelo para la promoción de vivienda pública.

Los trece restantes, a día de hoy, están pendientes de ser iniciados:

- PTS de Zonas canterables.

- PTS de Gestión de vertederos.

- PTS Rural vasco.

- PTS de Ordenación de los recursos turísticos.

- PTS de Carreteras de Guipúzcoa.

- PTS del Sistema aeroportuario.

- PTS de Puertos deportivos.

- PTS de Telecomunicaciones. 
- PTS de Ordenación de la distribución del gas

- PTS Hidrológico.

- PTS de Saneamiento de Aguas residuales.

- PTS de Eliminación de residuos.

- $\quad$ PTS de Equipamientos.

Por último, hay que reseñar que las directrices deben contar, por ley, con un periodo de vigencia de ocho años tras los cuales se procederá a su revisión. Sin embargo, el propio Gobierno Vasco, cada dos años, elabora una memoria denominada «Situación de las directrices de ordenación territorial» en la que se analiza su nivel de aplicación, su incidencia en el planeamiento territorial y municipal y las acciones a emprender para evitar los desajustes identificados. Esto hace que, tanto los planes territoriales parciales como los sectoriales, sigan estos mismos ritmos de vigencia y revisión.

A continuación se adjunta, de forma resumida, el contenido de dos planes territoriales sectoriales bien distintos. De esta manera, se puede comprobar la diferencia entre uno, el primero, con un carácter más neto de conservación y otro, el segundo, con un carácter más desarrollista.

\subsubsection{PTS Zonas Húmedas}

Lo primero que hay que tener en cuenta dentro de este PTS son los textos legales que han iluminado el trabajo. En este sentido, hay que destacar cuatro documentos básicos:

- Convenio de Ramsar (1971,1982).

- Convenio sobre la Biodiversidad (Río 1992).

- Directiva Hábitats (92/43/CEE).

- Directiva Aves (79/409/CEE).

Por otra parte, hay que tener en cuenta el marco jurídico que existe a nivel de la CAV. En este sentido, hay que destacar dos documentos legales importantes:

- Ley 90 de OT en el País Vasco.

- Ley 97 de Directrices de OT.

Es precisamente de esta última de la que se deriva y en la que ha bebido el presente PTS. Hoy en día, este plan se encuentra en fase de contestación a las alegaciones después de la fase de información pública. La previsión es que quedará aprobado para comienzos del 2004.

Como definición de lo que se entiende como zona húmeda, el Gobierno Vasco toma la misma que se acordó en Ramsar 1971: son aquellos terrenos inundados o saturados de agua con una frecuencia o duración suficiente como para albergar vegetación hidrófila y un conjunto de especies animales características de estos medios. Dentro de esta tipología general entran otras más particulares y con casuísticas muy variadas: marismas, pantanos, turberas, tremedales, brazos abandonados de ríos, charcas, lagunas, lagos, ibones, etc.

Se estima que en menos de 50 años, de contar con un territorio bajo estas condiciones de $72 \mathrm{~km}^{2}$ a nivel de País Vasco, se ha llegado en la actualidad a contar con $47 \mathrm{~km}^{2}$. Ha existido una merma o pérdida considerable, sobre todo por intervención del ser humano.

Existen una serie de objetivos generales que intentar conseguirse. Entre estos cabría destacar los tres primeros y más primordiales: 
- Garantizar, para cada zona, los valores naturales con los que cuenta.

- Posibilitar la mejora de las zonas húmedas degradadas.

- Establecer líneas de acción para revalorizar los recursos naturales.

Por otra parte, hay que destacar que este PTS llega a establecer una tipología clasificando bajo diferentes figuras las diferentes áreas inundadas. De esta forma existirían tres grandes grupos en los que estarían las A) Costeras, B) Interiores, C) Otras. Y, a su vez, estos tres grandes grupos son divididos en otros subgrupos, de manera que se hace una clasificación muy pormenorizada y detallada, intentando delimitar los ámbitos y las características fundamentales de cara al análisis, diagnóstico y creación de alternativas, más apegada al terreno posible.

Los impactos que estas zonas han sufrido han sido tradicionalmente los ligados al desarrollo urbano: rellenos antrópicos, urbanización, generación de infraestructuras, vertidos de escombros, polderización, drenaje forzado, extracción de áridos, caza, pesca, presión recreativa, etc.

Una vez analizados los impactos y cada uno de los sectores afectados por este plan, se realiza una clasificación de todos estos espacios de cara, sobre todo, a la ordenación y gestión apropiada de los mismos:

- GRUPO I: Afectados por la declaración de espacio natural protegido.

- GRUPO II: Humedales protegidos por planeamientos urbanísticos y los que se ordenan por el presente PTS.

- GRUPO III: El resto.

Una vez establecidos estos tres grandes grupos también se establecen las categorías de protección contempladas:

- Especial protección.

- Mejora ambiental (Restauración y recuperación).

- Protección del agua superficial (conservación de la calidad del agua).

- Zona agroganadera y de campiña (Mantenimiento de la capacidad agrológica).

- Forestal (Mantenimiento de usos y del bosque).

Posteriormente, se hace referencia a una serie de caracteres de las determinaciones, de manera que cada uno de los espacios inundados queda enmarcado por alguna de las figuras contempladas, teniendo en cuenta no sólo las del propio PTS, sino las categorías con las que aparecen dentro de los diferentes planes generales o normas subsidiarias a nivel local o municipal.

Por último, se hace referencia a las propuestas generales de actuación, un verdadero decálogo que, posteriormente vendrá acompañado con una serie de medidas más específicas para cada una de las categorías previas antes mencionadas. También se pasa a detallar algunos de los ejemplos. En este sentido, el PTS realiza un magnífico inventario de todas estas zonas y aplica para cada una un detallado programa de actuación teniendo en cuenta criterios como: el grado de conservación, las afecciones legales, el papel que ejercen, etc.

Las medidas generales o decálogo que se contempla tiene en cuenta las siguientes actuaciones:

- Elaboración de programas específicos.

- Actuaciones de protección. 
- Actuaciones de restauración.

- Actuaciones encaminadas al fomento y la regulación.

- Actuaciones con respecto al catastro.

- Aprobación de deslindes de dominio público.

- Adquisición pública de suelo.

- Inclusión de las zonas húmedas como zonas sensibles.

2.2.2. PTS Creación del suelo de actividades económicas y de equipamientos comerciales

Este PTS se basa en una serie de documentos legales que lo iluminan:

- $\quad$ Ley 4/1990 de O del T del País Vasco (L.O.T).

- Directrices de Ordenación Territorial del País Vasco (D.O.T.).

- Acuerdo programático del Gobierno Vasco (Legislatura 1999-2002).

Antes del presente ya existió otro PTS aprobado en 1994, y de hecho, éste ha sido el que ha venido actuando. Este es el que se pretende sustituir con el presente. El actual documento ya ha pasado el trámite de información pública y se encuentra preparado para su aprobación inicial.

Objeto: el suelo para todas aquellas actividades que lo demandan urbanizado en grandes cantidades:

- Plataformas logísticas

- Grandes centros comerciales

- Grandes zonas de almacenamiento y estocage.

Vocación: Generalista, se le ha dotado de un carácter más abierto y prospectivo y menos vinculante y normativo.

Se parte de una realidad compleja que contempla el territorio como soporte de las actividades económicas. Un territorio peculiar, muy accidentado en la vertiente cantábrica, donde el factor suelo cuenta con una gran importancia. De esta forma buscar y adecuar suelo se hace bastante difícil. Incluso ya se plantea la necesidad de crear un catálogo en el que se pueda inventariar dicho recurso. En la zona alavesa o toda la vertiente mediterránea, la disponibilidad es mayor y mejor.

Sin embargo, con los nuevos medios técnicos ya se comienzan a barajar posibilidades de generación de cualquier tipo de polígono en espacios con características complicadas como fuertes pendientes. Por lo tanto, se pueden contemplar nuevos criterios de actuación.

También se hace necesario ubicar estratégicamente los suelos y las actividades teniendo en cuenta factores como:

- Red de carreteras.

- Red ferroviaria.

- Puertos navales.

- Aeropuertos.

Otro factor importante es la localización de la población y el empleo. Hay que tender a ubicar los polígonos en aquellas zonas donde existan importantes concentraciones de población y donde ésta presente los niveles más altos de desempleo. 
Hay que tener en cuenta también la normativa derivada de cada uno de los planes urbanísticos o normas subsidiarias a nivel local o municipal. En este sentido, se ha ido realizando a esta escala un inventario del suelo urbanizado y el urbanizable disponible, para un futuro a medio y largo plazo. A modo de aclaración, como media, a nivel de la $\mathrm{CAV}$, cada año se ha venido consolidando un suelo que se corresponde con $180 \mathrm{Ha}$. Este es el ritmo que se ha venido manteniendo desde 1982.

Otro factor de primer orden a tener en cuenta es la inventariación de las intenciones del sector público en la intervención del suelo.

En lo que respecta a los objetivos que persigue el presente PTS, hay que reseñar que cuenta con uno de corte generalista: Instrumento de regulación del proceso de elaboración de los PTP, junto a dos objetivos más concretos que serían:

- La sistematización de criterios para la programación de las operaciones de creación pública de suelo.

- Bases para la ordenación territorial de los nuevos equipamientos comerciales y terciarios de grandes dimensiones.

El ámbito estratégico de actividades económicas sería el sistema o red de ciudades de la CAPV, con su red básica de transportes y comunicaciones. No obstante, sí hay indicadas una serie de zonas de actividad logística (Z.A.L.). Dentro de éstas estarían: el Bilbao metropolitano, el eje Donostia-Bajo Bidasoa y el Eje Vitoria-Gasteiz-Miranda. También se contemplan otras áreas estratégicas para la actividad económica. Dentro de éstas están: Núcleos importantes de población, Corredores de fondo de valle y Cabeceras municipales.

La gestión del suelo debe superar el marco municipal y se plantea ir a unos ámbitos de tratamiento territorial de rango superior, adecuados a las áreas funcionales o, en todo caso, sectores más concretos y puntuales donde se pueden contemplar bajadas de escala.

A continuación se detallan los criterios generales que han sido utilizados para la ordenación:

- Que el desarrollo de la actividad debe extenderse a todas las áreas funcionales. No debería existir una especialización.

- Pautas de complementariedad con estrategias de mejora medioambiental y desarrollo sostenible.

- Equilibrio interno de la población y el empleo en cada una de las áreas funcionales.

- Incentivación de la reconversión para nuevas actividades productivas de los suelos industriales obsoletos (hacia otros usos parecidos).

- Agilización de la capacidad de intervención por parte de la administración.

- Ponderación de la capacidad de desarrollo entre las diferentes áreas funcionales.

- Apuesta por un mestizaje de usos dentro de los mismos territorios, de manera que el ámbito vital y el del trabajo no se encuentren distanciados, de manera que se reduzca el tránsito de personas que colapsa las carreteras.

Una vez tenidas en cuenta estas medidas, se realiza una categorización del territorio con los ámbitos prioritarios y las áreas de incentivación. Se proponen tres categorías diferentes:

- Ámbitos de interés preferente.

- Ámbitos de crecimiento moderado.

- Ámbitos de bajo desarrollo. 
En lo referente a las acciones que deben realizarse dentro de las áreas de incentivación espacial, éstas se pueden resumir en varios puntos:

- Regeneración y reconversión de tejidos obsoletos.

- Política de incentivación económica en cabeceras comarcales, lejos de los grandes ejes de desarrollo (Encartaciones, Gernika-Bermeo, Markina, etc.)

- Dinamización de zonas agrícolas (Álava: Motaña, Valles, Laguardia, etc.).

Además se proponen una serie de actuaciones y propuestas de intervenciones:

- Condiciones de interés estratégico y público general con complementariedad y subsidiaridad con la iniciativa privada.

- Tipologías de intervención:

- Red de parques de actividades innovadoras (parques tecnológicos).

- Red de plataformas de servicios al transporte (plataformas logísticas).

- Red de reservas estratégicas (intervenciones puntuales).

- Carácter coyuntural de las actuaciones propuestas.

- Criterios de reparto y equilibrio territorial.

- Incidencia urbanística de los grandes equipamientos comerciales y centros de ocio:

- Ocupación de suelos estratégicos.

- Hiperdimensionamiento inadecuado.

- Deslocalización de los servicios de los núcleos urbanos.

- Propuestas de regulación territorial.

Como se observa, el presente PTS realiza una enorme labor de clasificación y de aplicación de un rosario de medidas adecuadas a cada una de las tipologías definidas con anterioridad.

\section{Fortalezas y oportunidades del territorio vasco}

Una vez determinada la base jurídica y la tipología completa de planificación territorial dentro del País Vasco, se van a apuntar cuales son las potencialidades o puntos fuertes y cuales las amenazas o puntos débiles que presenta esta región de cara a los próximos 16 años.

Para comenzar con las fortalezas y oportunidades, hay que reseñar que la primera viene dada como consecuencia de la existencia, precisamente, de este potente marco legal y de la buena instrumentalización de los contenidos de las leyes ya mencionadas. No sólo se debe valorar como positiva la configuración de una planificación a escala intermedia muy completa, y que aborda el territorio desde perspectivas globales, descendiendo después hacia ámbitos comarcales y locales, además de realizar pormenorizadas planificaciones a nivel sectorial, sino que además, esta variadísima tipología está siendo completada o, en el peor de los casos, aunque no completa se está a la expectativa de su realización en los próximos meses.

Otra de las grandes fortalezas del territorio es la existencia de una gran variedad de usos del suelo, de tipologías comarcales, de recursos, etc. Esto hace que no exista una gran especialización económica y territorial en unos sectores o hacia unos usos y vocaciones que, en un momento dado puedan entrar en crisis y terminar con el modelo propuesto. Muy al contrario, partiendo de una situación más especializada en un sector industrial pesado, se ha logrado superar una profunda crisis, remontar esa cierta especialización y variar tanto la economía como la vocación de las diferentes comarcas y municipios. 
Es importante tener en cuenta el dinamismo de la población que ocupa el territorio vasco. De hecho, existen importantes movimientos cooperativistas y una generación de autoempleo que esta siendo uno de los pilares básicos en la propia dinamización del territorio y en la captación de capitales e iniciativas foráneas. Todo ello partiendo de una situación política relativamente conflictiva, sufriendo los coletazos de una actividad violenta trasnochada y difícilmente justificable.

A parte de estas cuestiones, el nivel de vida, medido bajo diferentes aspectos como renta per capita, acceso a las nuevas tecnologías, oferta cultural, recursos medioambientales y de esparcimiento, capacitación laboral, poder adquisitivo, etc., es ciertamente alto, posiblemente una de las comunidades más adelantadas del estado español en todos estos parámetros. Esto viene acompañado, sin embargo, por un bajo índice de natalidad, de hecho, el País Vasco se configura como la región de Europa con cifras más bajas. Por lo tanto, se debe pensar en la necesidad de atraer población, no tanto del resto de las regiones vecinas, puesto que pasan por situaciones muy similares, sino de otras ubicaciones más alejadas e incluso fuera de la Unión Europea.

Otra oportunidad que muestra una gran potencialidad es la gran riqueza medioambiental, así como la variabilidad y calidad paisajística. Ahora se trata de regular perfectamente los usos a realizar dentro de las áreas mejor conservadas y contando con recursos como el paisaje, la fauna, la vegetación, las áreas costeras, las zonas inundadas, las áreas fluviales, etc. Muy unido a este filón de oportunidades se muestran las nuevas tendencias de ordenación. Se apuesta por un desarrollo sostenible y, al efecto, todos los documentos mencionados y descritos anteriormente deben estar imbuidos, en teoría, de la denominada «Estrategia ambiental vasca de desarrollo sostenible 2002-2020».

Este documento es un instrumento que el Gobierno Vasco ha aprobado este año en relación con el Medio Ambiente. Concretamente se aprobó el 4 de Junio de 2002, y fue elaborado como consecuencia de una preocupación al observar que la sociedad vasca vive en un contexto donde se demanda la ejecución de estrategias de estas características. La base de partida, a partir del contexto internacional, es un diagnóstico ambiental realizado en 2001 y que informa sobre la situación del propio País. Este diagnóstico pone de manifiesto que existen una serie de temas que habría que contemplar con mayor rigor, a saber:

- Consumo de recursos naturales.

- Emisión de gases con efecto invernadero y cambio climático.

- Emisión de productos químicos.

- Consumo exacerbado de suelo para generar zonas urbanas, etc.

Ante esta realidad, es necesario buscar herramientas para una desvinculación entre el crecimiento esperado de los agentes y los impactos ambientales. Es necesario integrar los objetivos de corte medioambientalista en todas las políticas sectoriales y dentro de la propia iniciativa privada.

Aunque se puede mostrar cierto grado de reticencia con respecto al cumplimiento de lo consensuado entre diferentes agentes sociales dentro de este documento, no deja de ser esperanzador como punto de partida. Las estrategias apuntadas en Río 1992, Posdam 1999 y la Estrategia Territorial Europea (ESDP), comienzan a calar e iluminar la ordenación a escalas intermedias y, con el tiempo, deberá configurarse como la alternativa necesaria si no quiere llegarse al colapso general del sistema.

Por otra parte, habría que reseñar el reto de seguir conservando los espacios con vocación agropecuaria. En este sentido, las producciones se van centrando en la unión del turismo, más o menos rural o ambiental, y la obtención de productos de calidad, con alto 
valor económico, tras diferentes etiquetaciones; denominación de origen, producto ecológico, label vasco, etc.

Para concluir, hay que reseñar que la idea de la Ciudad-Región vasca cuenta con una potencialidad ciertamente alta. Las oportunidades que pueden aparecer con el desarrollo sobre el terreno de este concepto no son pocas. Para ello, hace falta llegar a perpetuar un modelo muy similar a los ya existentes en Europa y cuyo paradigma puede ser el Ranstad holandés. Hace falta que la idea de la cooperación, de salvar las barreras culturales y territoriales provinciales en beneficio de un desarrollo más integrado, sostenible y basado en la cooperación, sea aceptado por la propia sociedad vasca en su conjunto. Esta idea podría servir para acabar con problemas internos como la falta de suelo en la vertiente atlántica, la obsolescencia de las grandes infraestructuras, etc. Por su parte, podría funcionar como un perfecto reclamo para la atracción de las iniciativas alóctonas y para conectar mejor el territorio con el resto de Europa.

\section{Debilidades y amenazas del territorio vasco}

Una vez observados los principales puntos fuertes, a través de las fortalezas y oportunidades que ofrece el territorio vasco, hay que tener en cuenta aquellos puntos más débiles, a través de las debilidades y amenazas más importantes, sobre todo con un carácter prospectivo, es decir, contemplando ese corto y medio plazo en el que se centran los diferentes planes.

La primera gran amenaza que aparece es la conexión de esta región con respecto al contexto europeo. En estos momentos se asiste al crecimiento de la Unión Europea y se vaticina, no sin razón, que el punto de gravedad de la propia Unión se va a ver alterado como consecuencia de la entrada de un buen número de países del este europeo. Ante este panorama cabe preguntarse hasta que punto puede ser importante una región que, como el resto del estado Español y Portugal, se hallan en una posición un tanto periférica y, con las nuevas incorporaciones, pierden peso específico, sobre todo a la hora de ser los destinatarios de los diferentes fondos estructurales y de las medidas de compensación y regularización de las que hace gala la ordenación a nivel europeo. A todo esto hay que añadir la propia disposición de Euskadi dentro del denominado «Eje Atlántico». En realidad este eje es más creído por las regiones que lo integran que por las políticas de desarrollo globales emanadas de la propia Unión. En este sentido, no deja de ser un eje secundario que, además, se encuentra con graves problemas de comunicación y conexión, debido a la insuficiencia notable de infraestructuras que terminen por vertebrar todas las regiones que lo integran.

Muy unido con este punto, la existencia de unas infraestructuras obsoletas y, en muchos casos, totalmente colmatadas, fundamentalmente de carreteras y ferrocarriles, hace que se esté perdiendo un precioso tiempo y que otras regiones puedan configurarse como bisagra entre la Península y el resto de Europa. Hasta la fecha, este papel ha sido el que, de momento, ha beneficiado de forma palpable al País Vasco. En esta línea, uno de los grandes problemas sigue siendo su especialización unívoca por el transporte de mercancías por carretera. De hecho, el ferrocarril no puede competir, hoy en día, ni en esta faceta ni en la de pasajeros. Este gran tráfico circula por las mismas vías que vertebran el tráfico interno, de manera que los colapsos son recurrentes y graves. A ello hay que sumar la grave situación de la red de carreteras guipuzcoanas, mientras en el resto del territorio vasco ya se han redactado los planes sectoriales pertinentes, en el caso mencionado todavía no se ha iniciado ni la redacción del preceptivo plan. Se siguen poniendo parches sin solucionar, de forma global, los problemas. 
En esta misma línea, autopistas como las que unen la frontera francoespañola con Bilbao y la que une esta última ciudad con Vitoria, siguen soportando peajes muy elevados, de los más caros de Europa, junto a Cataluña, de manera que continúan hipotecando el propio desarrollo vasco.

Otra de las grandes debilidades es la existencia de un reparto de competencias que no ayuda al desarrollo de todas las potencialidades recogidas anteriormente. De esta forma, el gran conflicto aparece con respecto a las competencias que todavía mantiene el estado. Aspectos como los puertos marítimos, los aeropuertos, los ferrocarriles, algunas carreteras, etc. siguen estando en manos del Gobierno Central. Un ejemplo claro es el de los ferrocarriles. Como se ha apuntado anteriormente, se requiere una profunda reestructuración del tráfico ferroviario, de hecho, no han existido inversiones de importancia desde los años 50 y el trazado ha quedado obsoleto y muy poco competitivo. Ante esto, el propio Gobierno Vasco pone en marcha el PTS ferroviario pero, al intentar desarrollar cuestiones como la implantación del tren de alta velocidad, se encuentra con que esta planificando sobre la nada, teniendo en cuenta que el consenso con el estado puede llegar a demorarse en el tiempo. Incluso en el caso de que se llegara a los acuerdos pertinentes, todavía no está clara la vocacionalidad del propio trazado de alta velocidad. De hecho, aunque se ha pretendido vender un tren con doble vocación; tránsito de mercancías y pasajeros, lo cierto es que el estado francés no parece muy dispuesto a que la conexión bajo su competencia cuente con esta misma vocacionalidad, con lo que el futuro es, cuando menos, incierto. A ello hay que sumarle el hecho de la falta de suelo para ubicar esta gran infraestructura y de si merece la pena un desembolso económico fuerte, una polémica compra de suelos y unos evidentes impactos ambientales si el resultado económico va a contar con graves incertidumbres.

En lo que respecta a una cuestión como es el modelo energético por el que se apuesta, existen importantes incógnitas de futuro. Aunque aparece una apuesta clara por las energías alternativas o renovables, sin embargo, se va a seguir dependiendo de dos fuentes de energía como son el petróleo (alóctona) y la electricidad (hasta cierto punto alóctona). No parece contar con mucho sentido el llenar de molinos de viento todas las cumbres de los montes vascos, con el consabido impacto ambiental y visual, mientras se sigue dependiendo mayoritariamente de las fuentes tradicionales.

Una de las grandes amenazas puede ser la gran presión que se ejerce sobre suelos con ciertos valores ambientales y aquellos que cuentan con la vocación agropecuaria. Esta es una realidad más palpable en la vertiente atlántica, por la densidad poblacional, lo compartimentado del relieve, la escasez de suelos con las suficientes garantías de edificabilidad, etc. El gran reto de la sostenibilidad viene de manos de la conservación de las zonas y valores ambientales, de la protección de la invasión de los márgenes fluviales, de las medias laderas dedicadas a la agricultura y ganadería, de las zonas húmedas, etc.

Otra de las grandes amenazas viene de la mano de la importación de un modelo territorial, social y económico anglosajón, fundamentalmente norteamericano. Desde este país llega la proliferación de las viviendas de baja densidad, la villa unifamiliar con jardín. Este modelo no se sostiene ante un territorio muy limitado, con una gran escasez de suelo. A ello hay que añadir el handicap que supone el dotar de infraestructuras a este hábitat tan disperso, a la vez que los tráficos por carretera aumentan y se da un menosprecio claro de los transportes colectivos. También en este sentido, se da la proliferación de las grandes superficies, con la pérdida de competencia del pequeño comerciante que tanto ha caracterizado, desde tiempos remotos, a la pequeña burguesía del país y al desarrollo que hoy en día tienen las ciudades y asentamientos de dicho territorio. Con todo ello se quiere comprar un modelo social donde la vida sea cada vez más individualista, se viva de forma autóno- 
ma, sin el continúo contacto con vecinos y amigos, modelo mediterráneo, más propio de culturas y territorios como el vasco y, en general, el peninsular.

Otra de las grandes debilidades, convertida en un enorme reto a superar, es la toma de conciencia y participación de los agentes sociales implicados en el proceso de ordenación. En lo que respecta a los entes más palpables; los ayuntamientos, éstos han visto que con la irrupción de la nueva ley del suelo, con la perdida de ciertas fuentes de financiación y, sobre todo, con la potente aparición de los diferentes documentos de ordenación de escala intermedia, han perdido un notable peso específico con el que contaban. Al respecto, existen importantes y continuas quejas y reclamaciones. No obstante, esta pérdida, en muchos casos, va a ser un aspecto positivo. Hasta ahora existía una ordenación del territorio desestructurada, a impulsos, donde los municipios más potentes se llevaban el gato al agua y podían llegar incluso a vetar las grandes líneas de ordenación si no venían a respetar o compartir las que presentaban los propios municipios. La oportunidad consiste en hacer entender a los ayuntamientos que se terminó el tiempo de la competencia, que es necesario dar lugar a procesos de ordenación más globales, coordinados, donde la cooperación se imponga en beneficio común.

Otro de los agentes sociales viene representado por los potentes e importantes grupos o agrupaciones vecinales y ciudadanas. A nivel del País Vasco existe un importante nivel asociativo aunque las autoridades se quejan amargamente de que sólo toman parte, dentro de los procesos de ordenación, en aquellos aspectos en los que se creen perjudicados. De esta manera, la participación sólo se da ante asociaciones de afectados y no tanto ante el gran público en general. Otro gran reto es la posibilidad de cambiar los medios y metodologías de consulta pública. De hecho, existen otros países donde las grandes líneas de ordenación se deciden por plebiscitos y, dependiendo de la escala, son decididas cuestiones como el modelo de desarrollo nacional, la red de infraestructuras que se requiere para un cantón o región, el tratamiento de los residuos que se quiere para un municipio o una mancomunidad, etc. Esto supone una toma clara e inequívoca de la responsabilidad de definir el modelo territorial y, por tanto, la ordenación y gestión del propio territorio. Si esta medida se desestima por cuestiones económicas o de organización, los diferentes grupos políticos podrían añadir en sus escuetos programas electorales todas estas cuestiones, de una forma más pormenorizada para que el ciudadano pudiera contar con un poder de resolución más directo y democrático.

Otro de los grandes agentes sociales es el ecologismo. En general, éste se queja porque no ve viabilidad al denominado desarrollo sostenible. Se observa como la conservación llega hasta los límites de los intereses económicos y éstos nunca tienen que retroceder ante un posible choque. Tampoco se está de acuerdo con modelos como el energético, el agroforestal, la recogida, depuración, eliminación, reutilización y reciclaje de los residuos, etc. Desde el ecologismo se piensa que no existe un modelo territorial global y claro y las actuaciones se restringen al taponamiento de las heridas más visibles, de forma que se va parcheando el territorio. Por otra parte, cuestiones como el tren de alta velocidad, la explotación de la energía eólica, etc. son proyectos con altos costes de inversión pública, con importantes impactos ambientales y con una dudosa viabilidad económica y social.

Otra de las debilidades es la existencia de un recurso como es el suelo, muy limitado, fundamentalmente en las grandes áreas urbanas. Esto, unido a los procesos especulativos derivados de este bien escaso, de la falta de escrúpulos de ciertos agentes, del incumplimiento de diferentes marcos legales y de la poca vigilancia del cumplimiento de las condiciones de la vivienda de oferta pública, hace que Donostia-San Sebastián se encuentre siempre entre las 3 ciudades con vivienda más cara del estado, Bilbao lo esté entre las 
8 primeras y Vitoria sea la ciudad que mayores crecimientos del precio del suelo ha registrado estos últimos 5 años.

Por último, existen escollos más puntuales que se centran en desequilibrios de diferente naturaleza entre las comarcas diferenciadas. Se da una notable distinción entre las más densamente pobladas y aquellas que menores densidades presentan pero, a su vez, existen otros desequilibrios dependiendo de cuestiones como; la escasez de infraestructuras, la despoblación, la incomunicación, la falta de suelos con garantías, la macrocefalia de algunos núcleos, los procesos especulativos, etc.

\section{Bibliografía}

GÓMEZ OREA, D. (2.002): Ordenación Territorial. Ediciones Mundi-Prensa (Agrícola Española S.A.). Madrid.

GOBIERNO DE ESPAÑA: Ley 6/1998, de 13 de abril, sobre régimen del suelo y valoraciones.

GOBIERNO DEL PAÍS VASCO: Ley orgánica 3/1979, de 18 de diciembre, Estatuto de Autonomía del País Vasco.

GOBIERNO DEL PAÍS VASCO: Ley 4/1990 de 31 de mayo, de Ordenación del Territorio del País Vasco.

GOBIERNO DEL PAÍS VASCO: Decreto 28/1997, de 11 de febrero por el que se aprueban definitivamente las Directrices de Ordenación Territorial de la C.A.P.V.

PUJADAS, R. y FONT, J. (1998): Ordenación y Planificación Territorial. Edit. Síntesis, Col. Espacios y Sociedades, Serie mayor № 8. Madrid.

ZOIDO, F. (1998): «Geografía y Ordenación del Territorio». Revista Íber, Didáctica de las ciencias sociales. Geografía e Historia, № 16, abril. Barcelona. Pp. 19 a 31.

La presente aportación se inscribe en el marco del proyecto: «Estrategias de Cooperación y Desarrollo Territorial Sostenible en el País Vasco y Navarra», dependiente del Ministerio de Ciencia y Tecnología. REF.: B502002-04233-C10-04. 